\title{
Development Trend of Human-Cyber Physical System in Manufacturing: Mixed Reality Patent Analytics
}

\author{
Amy J.C TRAPPEY, Dawi K. BAROROH ${ }^{\mathrm{a}, \mathrm{c}, 1}$, Halim B. SANTOSO ${ }^{\mathrm{b}, 1}$ and Armin \\ DARMAWAN $^{\mathrm{a}}$ \\ ${ }^{a}$ Department of Industrial Engineering and Engineering Management, National Tsing \\ Hua University, Hsinchu, Taiwan \\ ${ }^{\mathrm{b}}$ Institute of Service Science, National Tsing Hua University, Taiwan \\ ${ }^{\mathrm{c}}$ Department of Mechanical and Industrial Engineering, Universitas Gadjah Mada, \\ Yogyakarta, Indonesia
}

\begin{abstract}
Mixed Reality (MR) is an advanced technology designed to enhance users' experience and perception of interactions with objects in both virtual and real worlds. MR has enormous potential for improving human-cyber physical systems (HCPS) in the manufacturing industry. The transdisciplinary engineering (TE) characteristics of this research emphasize integrating advanced patent mining techniques to trend analysis of innovative MR technologies and applications in the manufacturing. Despite the growing popularity of MR applications, trend analyses in this emerging technology have relied on qualitative literature reviews and insufficient patent macro-analytics. Therefore, mapping patents related to MR inventions provides technology experts with better insights of technology development in the manufacturing. This research consists of a thorough review of non-patent literature (NPL) for the construction of MR domain ontology and a systematic patent mining analysis, including patent map analysis, patent clustering, patent topic modeling, and trend analysis of MR applications. A total of 709 patents from the InnovationQ Plus patent database are systematically identified and analyzed for trend discovery. The key terminologies, top ten patent assignees, top five applicants' country origins, top six Cooperative Patent Classifications (CPC), and seven technology clusters and topics are identified. These findings provide reliable MR-enabled HCPS suggestions for the manufacturing.
\end{abstract}

Keywords. Transdisciplinary, mixed reality, manufacturing, patent analytics, clustering, topic modeling, human-cyber physical system

\section{Introduction}

Global competition pushes companies to become innovative in their technological knowhow to enhance sustainable competitiveness in the marketplace. Due to the recent advances in information technologies (IT) and operational technologies (OT), the manufacturing industry has entered the era of Industry 4.0, equipped with so-called Cyber-Physical Systems (CPS). This revolution has increased productivity to meet the fast-growing consumer demands and support flexible and efficient manufacturing

\footnotetext{
${ }^{1}$ Corresponding Author, Mail: dawikb@gapp.nthu.edu.tw; halim.budi@iss.nthu.edu.tw.
} 
systems. On the contrary, some functions in the current manufacturing environment cannot be automated [1]. Therefore, allowing humans to collaborate with the CPS by playing "master" during manufacturing operations is a viable solution. The concept beyond CPS is considered the fifth industrial revolution, known as Human-Cyber Physical System (HCPS), as shown in Figure 1 [2]. HCPS is a type of composite intelligent system that combines humans, cyber systems, and physical systems to achieve specific goals at a high level of efficiency.

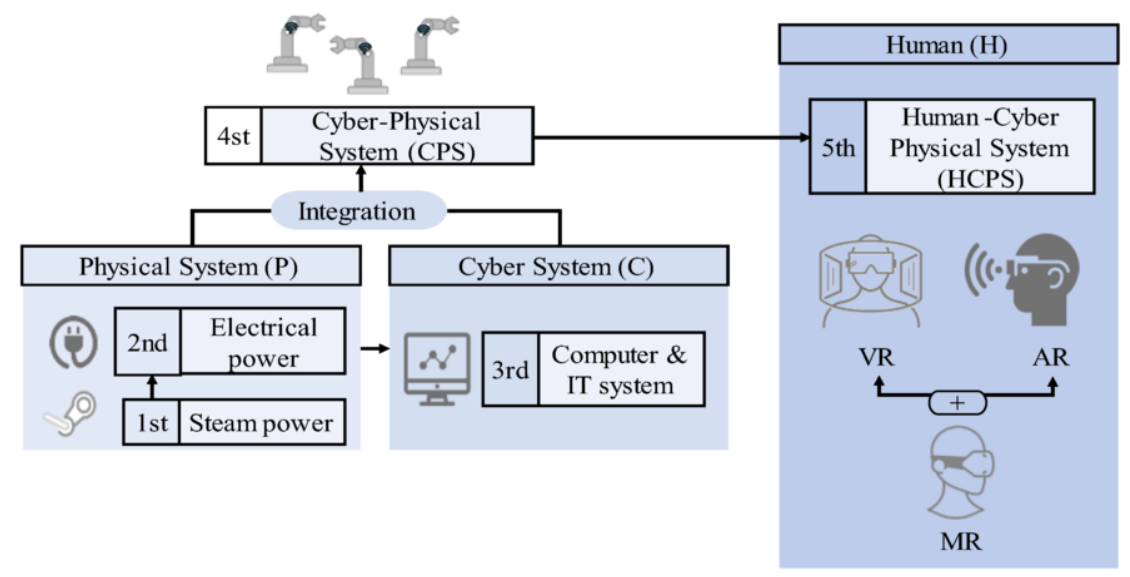

Figure 1. Human-CPS for advanced manufacturing (Industry 4.0).

Immersive technology is one of the best strategies to increase human involvement and realize HCPS in the manufacturing system. There is a taxonomy for this technology, which was developed by Milgram \& Kishimo [3]. Table 1 describes differences among Virtual Reality (VR), Augmented Reality (AR), and Mixed Reality (MR) [4] [5]. As we can see in table 1, MR is a combination between VR and AR. MR enhances user's perceptions and allows near-seamless interactions between objects in the virtual and real worlds. Information overlay enables remote collaborators to annotate the user's view to improve communications between collaborators. Therefore, MR has vast potential for realizing HCPS for smart manufacturing. To discuss MR technology and its implementation in different sectors briefly, we draw MR ontology based on the prior literature as shown in Figure 2.

This research depicts the MR technologies and applications using patent analytics as a tool for comprehensive technology mining. Technology mining helps discover stateof-the-art technology trends based on previous knowledge documents. Technology mining, adopting supervised and unsupervised learning, is considered an exercise of transdisciplinary engineering (TE), which provides complex engineering solutions by integrating a broad set of knowledge for practical problems [6]. Prior technology mining studies in the TE context are also found in [7].

Table 1. Classification of immersive technologies.

\begin{tabular}{lccc}
\hline Category & VR & AR & MR \\
\hline Immersive level & High & Low & Medium \\
\hline User Interaction & Low & High & Medium \\
\hline Environment & Virtual & Real & Mixed \\
\hline
\end{tabular}


This research focuses on the patent analysis of MR technologies and applications, mainly for the manufacturing industry. This study tries to fill the research gap by doing technology mining of MR in manufacturing to (1) highlight current technological trends that impact their respective application areas and (2) identify key technologies for drawing exciting research directions to support HCPS ideas. This study contributes to understanding MR's use in HCPS and manufacturing and gives the future research direction. The remainder of this paper is organized as follows. Following this introduction, the research methodologies are discussed. We will describe patent mining's analytical results in section 3 and conclude our study in the last section.

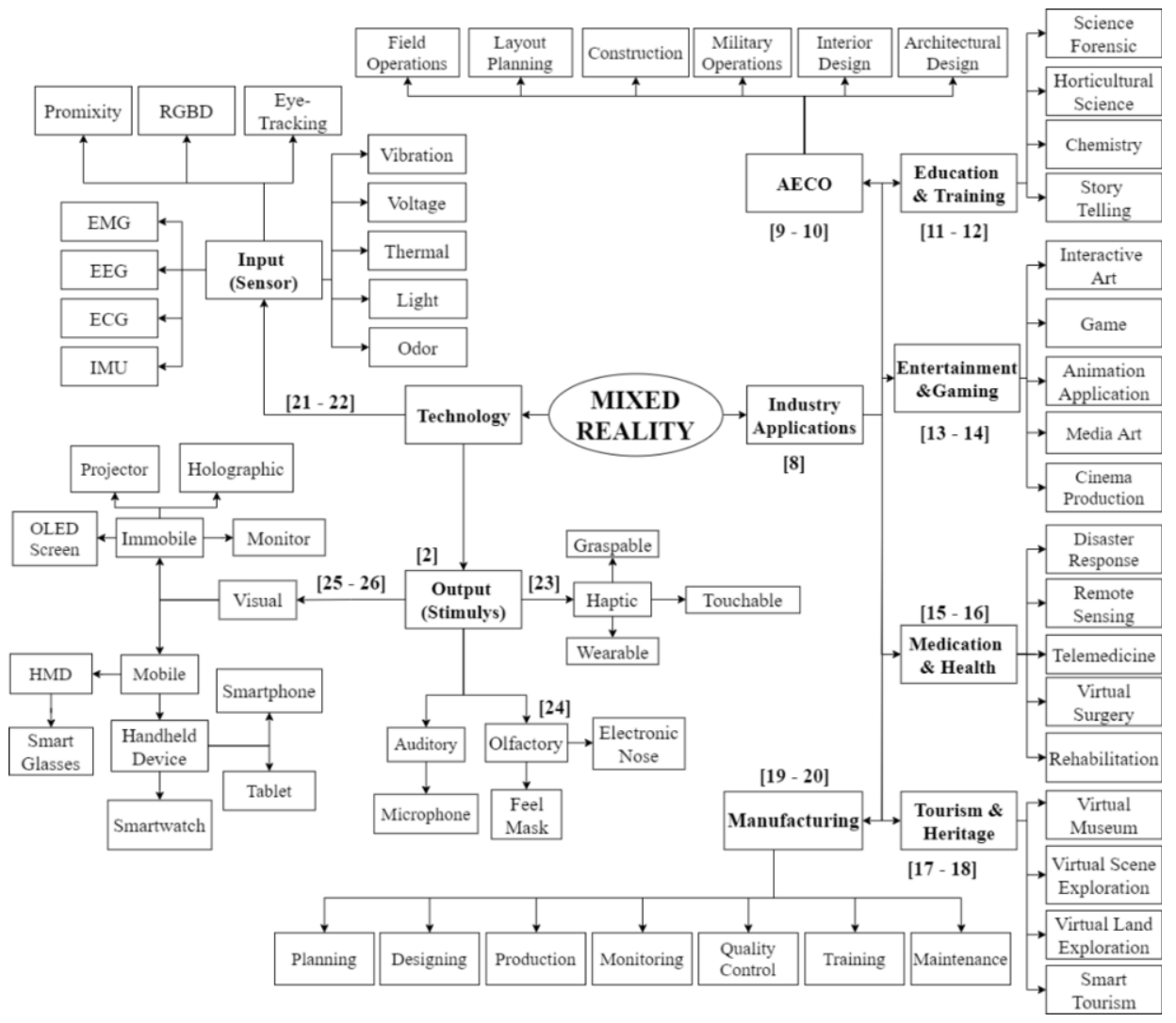

Figure 2. Mixed reality ontology-based literature review [2], [8]-[26].

\section{Research methodology}

We proposed technology mining based on patent analytics for this research, as illustrated in Figure 3, including technological specification and patent landscape. In the technological specification, we discuss the patent portfolio of MR in manufacturing. For patent landscape, clustering and each cluster's topic modeling (LDA) of domain patents are depicted. At the end of this section, we discuss the MR patent portfolio and its connections to HCPS implementations in the manufacturing industry. 


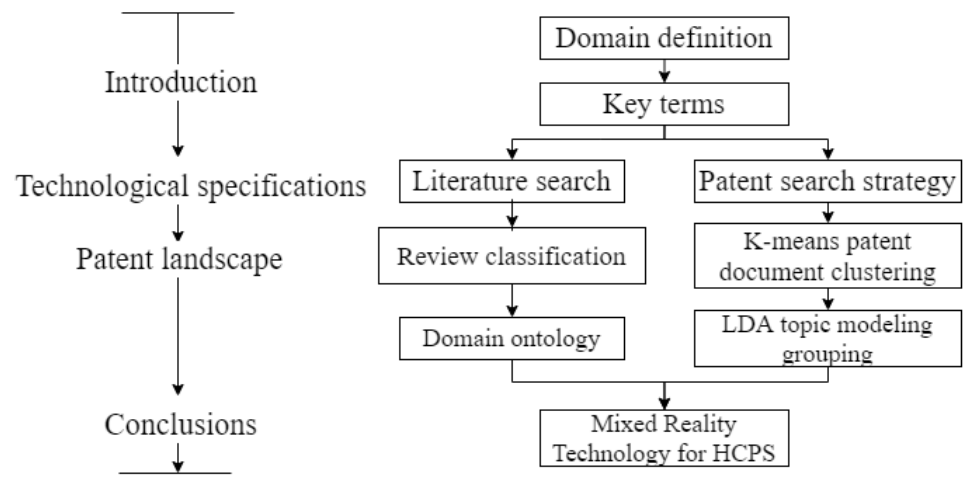

Figure 3. The research flow of comprehensive patent analytics.

\subsection{Technological specification}

This research analyzes patent literature by utilizing the InnovationQ Plus database from the last ten year (2010-2019) in different patent offices. Our keywords for this patent search are MR in Manufacturing in specific Cooperative Patent Classifications (CPC) (see Table 2 for more details). This study focuses on active and granted patent publication. Using the search strategy, we found 48,900 patent documents. We scan and read through those patent documents and choose patents that are mainly related to our topics. In the end, 709 patent documents are extracted and used for further analysis.

Table 2. Patent search strategy.

\begin{tabular}{|c|c|c|}
\hline No & Criteria & Value \\
\hline 1 & Database & InnovationQ-Plus \\
\hline 2 & Keywords & Mixed Reality in Manufacturing \\
\hline 3 & CPC Codes & $\begin{array}{l}\text { Including: G01B; G01C; G01D; G01R; G01S; G02B; G02F; G03H; G05B; } \\
\text { G05D; G06F; G06K; G06Q; G06T; G07F; G08B; G09B; G09G; G10L }\end{array}$ \\
\hline 4 & Office Patent & $\begin{array}{l}\text { All patent offices, including USPTO (United States), China (CNIPA), } \\
\text { World Intellectual Property Organization (WIPO), Taiwan (TIPO), } \\
\text { European (EPO), Japan (JPO), and Korea (KIPO) }\end{array}$ \\
\hline 5 & $\begin{array}{l}\text { Publication } \\
\text { Year }\end{array}$ & 1 January 2010 - 31 December 2019 \\
\hline 6 & Enforceability & Active and Granted \\
\hline
\end{tabular}

\subsection{Patent landscape}

In this subsection, we investigate our patent portfolio and briefly discuss three points: (1) top ten assignees; (2) five largest countries that contribute most to the technology development; (3) six CPCs mainly used in our portfolio. Our analysis's next step is by conducting K-means to cluster patent documents with similar technical specifications into a single group. In this clustering, we extracted some basic key terms from patent abstracts and claims. Based on those key terms and supported by the title of some patents, we named each cluster. From the technology cluster, we do topic modeling to explore 
the current topics for each cluster. We used Latent Dirichlet Analysis (LDA), an information retrieval algorithm, by considering topics as multinomial distributions over the words [27]. LDA is commonly used to collect words and topics from the combined publications, standards, and patent documents.

\section{Patent mining results and discussions}

\subsection{Patent map analysis}

Patent map analysis was developed based on a patent database from InnovationQ Plus. There are about 709 patent-related MR in manufacturing, as captured in Figure 4. First introduced in 1994 by Paul Milgram, patent publication related to MR in manufacturing experienced a linear increase until 2019. A significant increase was happened since the last three years ago; at least 406 (53\%) patent documents were published in this range of time. Generally, this trend means there is enormous potential to be involved in this area of MR in Manufacturing.

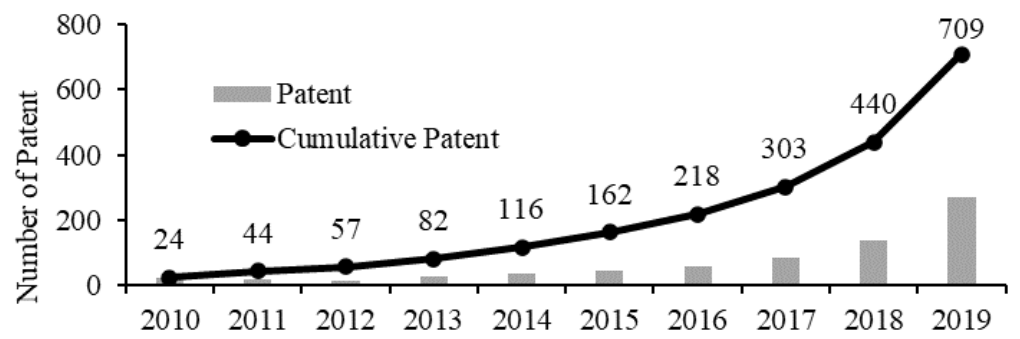

Figure 4. Numbers of patent publications per year (2010 - 2019).

Microsoft Tech Licensing still dominates the current assignee contributed about 131 patents document then followed by Canon and Ricoh from Japan (90 patents documents). These three companies contributed about 31\%-Samsung from Korea in fourth place with 13 patents. Simultaneously, Apple Inc and Intel Corp were in the fifth and sixth positions with 11 documents each. Nine publications were contributed by Electronic \& Telecom Research Institute, Facebook Tech LLC, and Magic Leap. Nokia is the only company from Finland in the last line with eight documents.

Regarding Figure 5, the US is still superior, with about $49 \%$ for patent publication MR in manufacturing. The top three Asia countries (Japan, Korea, and China) and Europe (represented by Germany) followed the US for their significant contributions. Japan contributes $21 \%$ of documents through its assignee and then followed by Korea $(12 \%)$ and China (11\%), respectively. Lastly, Germany contributed two percent.

Generally, most companies involved in a patent publication related to MR focus on creating a technology application concerned with 3D model manipulation for computer graphics, interface arrangement for data processing interacted with the human body, and optical systems apparatus. Based on Figure 5, the CPC scheme G06T concerned with manipulating 3D models for computer graphics using MR is the most dominant patent publication. About 224 documents were published with CPC G06T schemes. Furthermore, in the second row, 133 patents published the CPC Scheme G06F concerned with input arrangement for transferring data from processing in and out with human body interaction. G02B (CPC scheme for optical systems and apparatus characterized by 
visual features) in the third row with 100 patent publications. Data recognition (G06K), about 30 patent publications followed in the fourth. G06Q Data Processing System, with 29 patent publications, existed in fifth. Lastly, H04N related Pictorial Communication Scheme with 29 documents.

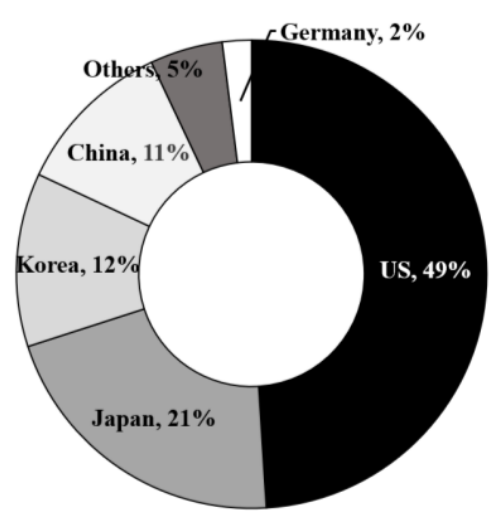

(a)

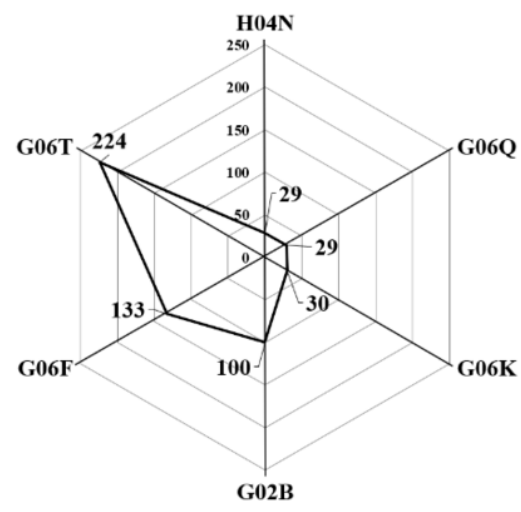

(b)

Figure 5. Patent analysis based on (a) country of origin and (b) cooperative patent classifications.

\section{2. $\quad K$-means for patent document clustering}

We found seven technology clusters related to mixed reality in manufacturing. Most documents are in the OLED Screen and Holographic Technology cluster due to its early development and needs. While the technology generally causes minor documents in Smell and Taste Sensory Technology cluster, it is still only needed in a specific manufacturing industry. The rest distribution of the patent document can be accessed in Table 3 .

Top words representative in each cluster were identified in the clustering process. Figure 6 shows the top words and key phrases for every cluster. In the auditory technology cluster (cluster 0 ), there are key phrase representatives related to the audio, signal, channel audio, audio track, and audio source. For the OLED screen technology cluster (cluster 2), five words are the most representative words related to technology equipment (screen, apparatus display, liquid crystal, electronic display, and crystal display. The holographic technology cluster (cluster 3 ) has five topic key phrases related to tracking position and object (holographic object, object distance, space physical, space object, and space superimpose). The haptic technology cluster (cluster 4) has six keywords: stylus, touch, glove, tactilely detectable, and electromagnetic wave. In the eye-tracking technology cluster (cluster 5), five keywords correspond to sensing using head movement/ gaze (eye content, eye display, eye position, eye gaze, and pupil position). Then, for the HMD technology cluster (cluster 6), the conductive material, biosignal, sensor indicate, signal sensor, and synthetic object are five key phrases of the HMD cluster. The last, the smell \& taste sensory technology cluster (cluster 6), has the head mount, gaze ray, geo-locate, position head, and plurality head. 
Table 3. The patent document clustering result.

\begin{tabular}{lll}
\hline Cluster number & Cluster name & The number of patents \\
\hline 1 & Auditory Technology Cluster & 142 \\
\hline 2 & OLED Screen Technology Cluster & 155 \\
\hline 3 & Holographic Technology Cluster & 153 \\
\hline 4 & Haptic Technology Cluster & 61 \\
\hline 5 & Eye-tracking Technology Cluster & 76 \\
\hline 6 & HMD Technology Cluster & 86 \\
\hline 7 & Smell \& Taste Sensory Technology Cluster & 36 \\
\hline
\end{tabular}

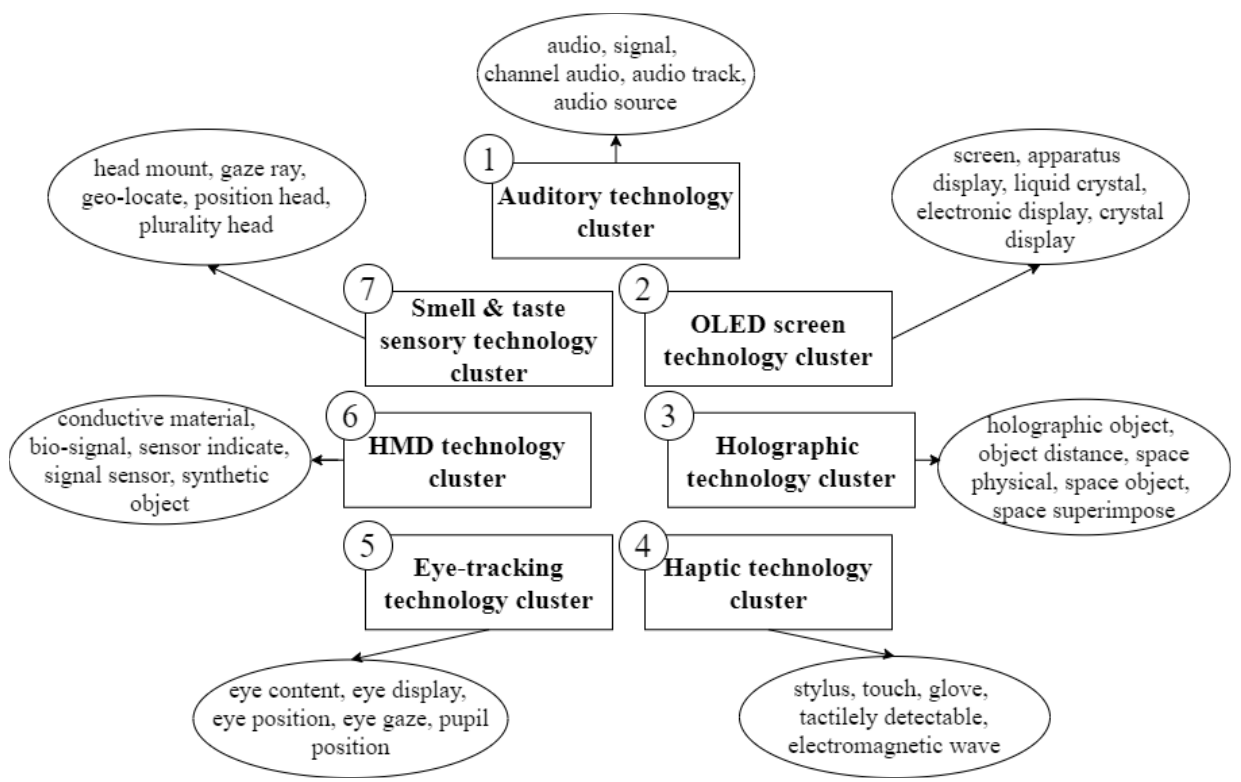

Figure 6. Technology cluster for mixed reality in manufacturing.

\subsection{LDA patent topic modeling}

Based on the simulation schemes, there is the optimum number of the topic for each cluster namely four topics for cluster 1 (auditory), three topics for cluster 2 (OLED screen), three topics for cluster 3 (holographic), three topics for cluster 3 (haptic), two topics for cluster 5 (eye-tracking), and three topics for cluster 6 (HMD). Unlike other clusters, for cluster 6 (smell \& taste), we did similarity of top words, and we found that from 2 topic groups, the similarity of 30 top words is around $90 \%$. Therefore, we merge both topics to become only one topic. These results can be seen in Table 4 . 
Table 4. List of topics for each cluster technology.

\begin{tabular}{|c|c|c|c|}
\hline Cluster & Title & Topic & Words \\
\hline \multirow[t]{4}{*}{1} & \multirow[t]{4}{*}{ Auditory } & Detection System & Detection, Match, Threshold, Pose, Pattern \\
\hline & & Recognition System & Monitoring, Process, Flow, Condition, Scene \\
\hline & & Retrieval System & Information, Retrieve, Selectable, Readable, Audio \\
\hline & & Storage System & Audio, Signal, Sound, Storage \\
\hline \multirow[t]{3}{*}{2} & \multirow[t]{3}{*}{ OLED Screen } & Stereoscopic System & Stereoscopic, Imaging, Processing \\
\hline & & Apparatus System & Polarizing, Reflective, Layer \\
\hline & & $\begin{array}{l}\text { Visualization } \\
\text { System }\end{array}$ & Visualization, Representation, Environment \\
\hline \multirow[t]{3}{*}{3} & \multirow[t]{3}{*}{ Holographic } & Data Processing & $\begin{array}{l}\text { Input, Orientation, Viewpoint, Physical, Dimensional, } \\
\text { State }\end{array}$ \\
\hline & & Stereoscopic System & Superimpose, Color, Presentation, Extract \\
\hline & & Apparatus System & Processing, Video, Graphic, Projector \\
\hline \multirow[t]{3}{*}{4} & \multirow[t]{3}{*}{ Haptic } & Gesture Tracking & Tracking, Object, Marker, Angle, Hand \\
\hline & & Media Interface & Hardware, Interface, Apparatus, Equipment, Mobile \\
\hline & & $\begin{array}{l}\text { Display } \\
\text { Architecture }\end{array}$ & Refractive, Curvature, Radius, Characterize \\
\hline \multirow[t]{2}{*}{5} & \multirow[t]{2}{*}{ Eye-Tracking } & Object Recognition & Recognition, Text, Object, Marker \\
\hline & & Eye/ Head Tracking & Gaze, Eye, Direction, Pupil \\
\hline \multirow[t]{3}{*}{6} & \multirow[t]{3}{*}{ HMD } & Object Tracking & $\begin{array}{l}\text { Tracking, Object, Movement, Distance, Direction, } \\
\text { Visual }\end{array}$ \\
\hline & & Data Processing & Processing, Information, Content, Visual \\
\hline & & Object Rendering & Rendering, Object, Surface \\
\hline 7 & $\begin{array}{l}\text { Smell \& } \\
\text { Taste Sensory }\end{array}$ & Apparatus System & Feedback, Information, Apparatus, Sense \\
\hline
\end{tabular}

\subsection{MR technology for human-CPS}

Some interesting findings were derived from the patent analysis described in the previous section. We elaborate on these findings by providing possible explanations for technology mining, especially MR technology in manufacturing operations, to support the human-cyber physical system (HCPS). First, MR is the best immersive technology that creates a world where humans and machines interact seamlessly. The number of patent technology of MR is increasing year to year. However, there is a challenge for MR development, especially to make it more human-friendly [2] [4] [28]. Technology mining of patent analysis in MR technology and application is the best representative of the TE approach for accommodating engineering solutions in practice. Second, developing MR technologies still focus on manipulating 3D models, optical systems and apparatus, and data recognition \& processing. Humans have some limitations, such as 
the human eye limitation for display, which can only be placed 3 to $4 \mathrm{~cm}$ away from the eye. It makes optical sensing and processing crucial elements for immersive systems [28]. Third, we got seven technology clusters from k-means analysis, but most of them use visual perception as the primary communication channel. Although visual clues are the most effective for discriminating between con- and hetero-specific individuals, excessive visual stimuli can distract human receivers. Therefore, creating the MR technologies which support multi-modality can be a practical approach for improving information dissemination or overcoming limitations in a real manufacturing environment. Thus, creating multisensory experiences is the dominant direction [2] [15] [16]. Last, based on LDA analysis, MR technologies emphasize apparatus, system, and method to deal with recognition, processing, and visualization. Some manufacturing operations still involve hand operations and human operators' decision-making [2] [5]. It requires close attention and awareness from human operators to identify the related cues or signals from the manufacturing environment. MR helps increase human contextawareness in real-time with a reduced mental workload, which can help prevent human errors and support the critical goal of HCPS.

\section{Conclusions}

MR enhances the Cyber-Physical System (CPS) through human collaboration and involvement with the intelligent machines, known as Human-Cyber Physical System (HCPS). Technology mining enhances the understanding of state-of-the-art MR technology development. MR technologies and applications are still emerging topics in manufacturing fields, as indicated by the growing patent literature in the past ten years (2010 - 2019). Most technology companies, such as Microsoft, Samsung, and Apple, dominate MR technology developments and their innovative applications. We extend our study by providing a technology cluster of MR and discovering critical topics related to those clusters by utilizing unsupervised clustering and topic modeling approaches. Visual perception is dominant in the MR patent landscape. Innovative sensory technologies, such as auditory, haptic, smell, and taste, are still being researched to bring an immersive environment for HCPS in various manufacturing sectors.

\section{References}

[1] J. Manyika, A future that work: Amazing progress in AI and Automation, McKinsey Glob. Inst. Res., no. June, 2017.

[2] D. K. Baroroh, C.-H. Chu, and L. Wang, Systematic Literature Review On Augmented Reality In Smart Manufacturing: Collaboration Between Human And Computational Intelligence, J. Manuf. Syst., pp. 1-16, 2020, [Online]. Available: https://doi.org/10.1016/j.jmsy.2020.10.017.

[3] P. Milgram and F. Kishimo, Taxonomy of Mixed Reality Visual Displays, IEICE Trans. Inf. Syst., vol. 77, no. 12, pp. 1-15, 1994.

[4] S. Rokhsaritalemi, A. Sadeghi-Niaraki, and S. M. Choi, A review on mixed Reality: Current trends, challenges and prospects, Appl. Sci., 2020, Vol. 10, no. 636, pp. 1-26, DOI: 10.3390/app10020636.

[5] G. Riexinger, A. Kluth, M. Olbrich, J. D. Braun, and T. Bauernhansl, Mixed Reality for On-Site SelfInstruction and Self-Inspection with Building Information Models, Procedia CIRP, 2018, Vol. 72, pp. 1124-1129, DOI: 10.1016/j.procir.2018.03.160.

[6] A. Kharlamov, G. Parry, and L. Newnes, When and where is transdisciplinary engineering applied in projects? A case study, Advances in Transdisciplinary Engineering, 2019, vol. 10, pp. 12-21.

[7] A. J. C. Trappey, C. V. Trappey, C. Y. Fan, and I. J. Y. Lee, Mining the customer's voice and patent data for strategic product quality function deployment, Advances in Transdisciplinary Engineering, 2017, Vol. 5, pp. 985-992, DOI: 10.3233/978-1-61499-779-5-985.

[8] R. A. J. de Belen, H. Nguyen, D. Filonik, D. Del Favero, and T. Bednarz, A systematic review of the 
current state of collaborative mixed reality technologies: 2013-2018, AIMS Electron. Electr. Eng., vol. 3, no. 2, pp. 181-223, 2019, DOI: 10.3934/ElectrEng.2019.2.181.

[9] W. Huang et al., SMART: Design and evaluation of a collaborative museum visiting application, International Conference on Cooperative Design, Visualization and Engineering, 2015, Springer, Cham, DOI: 10.1007/978-3-319-24132-67.

[10] P. Kallioniemi et al., Evaluating landmark attraction model in collaborative wayfinding in virtual learning environments, Proceedings of the 12th International Conference on Mobile and Ubiquitous Multimedia, 2013, DOI: 10.1145/2541831.2541849.

[11] D. Aschenbrenner, M. Li, R. Dukalski, J. Verlinden, and S. Lukosch, Collaborative Production Line Planning with Augmented Fabrication, 25th IEEE Conference on Virtual Reality and $3 D$ User Interfaces, VR 2018 - Proceedings, 2018, pp. 509-510, DOI: 10.1109/VR.2018.8446533.

[12] P. Galambos et al., Design, programming and orchestration of heterogeneous manufacturing systems through VR-powered remote collaboration, Robot. Comput. Integr. Manuf., 2015, DOI: 10.1016/j.rcim.2014.08.012.

[13] L. Peppoloni, F. Brizzi, E. Ruffaldi, and C. A. Avizzano, Augmented reality-aided tele-presence system for robot manipulation in industrial manufacturing, Proc. ACM Symp. Virtual Real. Softw. Technol. VRST, vol. 13-15-Nove, pp. 237-240, 2015, DOI: 10.1145/2821592.2821620.

[14] D. Borrmann, F. Leutert, K. Schilling, and A. Nüchter, Spatial projection of thermal data for visual inspection, 2016 14th Int. Conf. Control. Autom. Robot. Vision, ICARCV 2016, vol. 2016, no. November, pp. 13-15, 2017, DOI: 10.1109/ICARCV.2016.7838617.

[15] H. Culbertson, S. B. Schorr, and A. M. Okamura, Haptics: The Present and Future of Artificial Touch Sensation, Annu. Rev. Control. Robot. Auton. Syst., 2018, DOI: 10.1146/annurev-control-060117105043.

[16] J. Wang, J. Erkoyuncu, and R. Roy, A Conceptual Design for Smell Based Augmented Reality: Case Study in Maintenance Diagnosis, Procedia CIRP, 2018, Vol. 78, pp. 109-114, DOI: 10.1016/j.procir.2018.09.067.

[17] W. Fang, L. Zheng, H. Deng, and H. Zhang, Real-time motion tracking for mobile augmented/virtual Reality using adaptive visual-inertial fusion, Sensors (Switzerland), 2017, Vol. 17, no. 5, DOI: 10.3390/s17051037.

[18] J. Wang, Y. Feng, C. Zeng, and S. Li, An augmented Reality based system for remote collaborative maintenance instruction of complex products, IEEE Int. Conf. Autom. Sci. Eng., vol. 2014-Janua, pp. 309-314, 2014, DOI: 10.1109/CoASE.2014.6899343.

[19] A. Shekhar Nittala, N. Li, S. Cartwright, K. Takashima, E. Sharlin, and M. C. Sousa, PLANWELL: Spatial user interface for collaborative petroleum well-planning, In SIGGRAPH Asia 2015 Mobile Graphics and Interactive Applications, 2015, DOI: 10.1145/2818427.2818443.

[20] A. R. Singh, V. Suthar, and V. S. K. Delhi, Augmented reality (AR) based approach to achieve an optimal site layout in construction projects, ISARC 2017 - Proc. 34th Int. Symp. Autom. Robot. Constr., no. February 2018, pp. 165-172, 2017, DOI: 10.22260/isarc2017/0022.

[21] S. Fleck and G. Simon, An augmented reality environment for astronomy learning in elementary grades: An exploratory study, In Proceedings of the 25th Conference on l'Interaction HommeMachine, 2013, DOI: 10.1145/2534903.2534907.

[22] I. M. Gironacci, R. Mc-Call, and T. Tamisier, Collaborative Storytelling Using Gamification and Augmented Reality, In International Conference on Cooperative Design, Visualization and Engineering, 2017, DOI: 10.1007/978-3-319-66805-5_12.

[23] E. Ch'ng, D. Harrison, and S. Moore, Shift-life interactive art: Mixed-reality artificial ecosystem simulation, Presence Teleoperators Virtual Environ., 2017, DOI: 10.1162/PRES_a_00291.

[24] M. McGill, J. H. Williamson, and S. Brewster, Examining the role of smart TVs and VR HMDs in synchronous at-a-distance media consumption, ACM Trans. Comput. Interact., 2016, DOI: $10.1145 / 2983530$.

[25] G. Kurillo, A. Y. Yang, V. Shia, A. Bair, and R. Bajcsy, New emergency medicine paradigm via augmented telemedicine, in International conference on virtual, augmented and mixed reality, 2016, DOI: 10.1007/978-3-319-39907-2_48.

[26] L. A. Shluzas, G. Aldaz, and L. Leifer, Design thinking health: Telepresence for remote teams with mobile augmented Reality, in: H. Plattner, C. Meinel, L. Leifer (eds.) Design Thinking Research: Making Design Thinking Foundational, 2015, pp. 53-66.

[27] U. H. Govindarajan, A. J. C. Trappey, and C. V. Trappey, Intelligent collaborative patent mining using excessive topic generation, Adv. Eng. Informatics, 2019, Vol. 42, no. January, p. 100955, DOI: 10.1016/j.aei.2019.100955.

[28] U. H. Govindarajan, A. J. C. Trappey, and C. V. Trappey, Immersive Technology for Human-Centric Cyberphysical Systems in Complex Manufacturing Processes: A Comprehensive Overview of the Global Patent Profile Using Collective Intelligence, Complexity, vol. 2018, 4283634. 